\title{
A power-efficient synchronization scheme for the MB-OFDM-based UWB systems
}

Xiu-Wen Yin ${ }^{1,2}$ and Hong-Zhou Tan ${ }^{1,2^{*}}$

\begin{abstract}
Due to the complexity of multi-band orthogonal frequency-division multiplexing (MB-OFDM), achieving low power is still one of the major difficulties in designing the MB-OFDM-based ultra wideband (UWB) receiver. This paper targets at providing a very low power synchronization scheme with high performance for the MB-OFDM UWB receiver. First, the power consumptions of the individual functions are analyzed based on the overall architecture of the scheme. Then, the individual functions are designed. A time-frequency code (TFC) identification scheme is proposed, which divided the TFC identification process into two steps. A signal detector (SD) is designed for the first step to judge whether preamble symbols are present, and a TFC type detector (TTD) is designed for the second step to identify the TFC type. A packet detector is proposed, in which a coarse detector (CD) and a final detector (FD) are designed for reducing power consumption and improving performance, respectively. Also, a matched filter is proposed to simplify the correlation operation between the received symbol and the preamble symbol. The evaluation results show that the proposed TFC identification scheme and the packet detector have obvious lower computational complexity compared to existing solutions. The results also show the TFC identification scheme has satisfactory performance and that the performance of the packet detector is excellent.
\end{abstract}

Keywords: MB-OFDM; UWB; Synchronization; Low power

\section{Introduction}

High-speed wireless communication through wireless personal area network (WPAN) can bring great convenience to users. Ultra wideband (UWB) technology can offer a solution for the data rate, quality of service (QoS) and power consumption requirements of the next-generation high-speed WPAN due to features such as wide bandwidth, low interference from other wireless systems, and low power density. Compared to another UWB technique, impulse radio (IR)-UWB, the multi-band orthogonal frequency-division multiplexing (MB-OFDM) has higher spectrum efficient and better robustness in dispersive channel and is more suitable for high data rate transmission. Due to these good features, the MB-OFDM has been adopted by many groups such as Wireless USB Promoter Group.

The Federal Communications Commission (FCC) has allocated 7,500 MHz (3.1 to $10.6 \mathrm{GHz}$ ) spectrum for UWB

\footnotetext{
* Correspondence: issthz@sysu.edu.cn

${ }^{1}$ School of Information Science and Technology, Sun Yat-sen University, Guangzhou 510006, China

${ }^{2}$ SYSU-CMU Shunde International Joint Research Institute, Foshan 528300, China
}

devices. In MB-OFDM UWB, The entire 7,500 $\mathrm{MHz}$ spectrum is divided into five band groups and fourteen $528 \mathrm{MHz}$ bands [1]. During data transmission, a timefrequency code (TFC) is used for frequency hopping among the bands that of the same band group [1,2].

Due to high activity, the synchronization block is one of the major power consumption sources in the MBOFDM UWB receiver. To design the synchronization block with low power consumption is still one of the major challenges in the design of the MB-OFDM UWB receiver. Also, OFDM-based systems are very sensitive to timing and carrier frequency offset. Synchronization errors cause inter-symbol interference (ISI) and inter-carrier interference (ICI), which destroy the orthogonality of the OFDM subcarriers and result in severe system performance degradation.

The synchronization design for OFDM-based systems has been researched for years. Many methods have been proposed for normal OFDM systems [3-7]. Although some experiences of normal OFDM systems can be developed for MB-OFDM UWB systems, most methods for normal OFDM systems cannot be used for the MB-OFDM UWB 
system directly due to some special features of MB-OFDM UWB such as the frequency hopping and low power spectral density (PSD). By considering the special features of MB-OFDM UWB, many effective methods have been proposed specially for MB-OFDM UWB systems [8-18]. A cross-correlation (CC)-based method is proposed in [8] for TFC identification. Some methods [10-12,14] are proposed for packet detection. In [11], a sign-bit CC algorithm is proposed to simplify the operation of the normal CC. The sign-bit CC algorithm is further simplified in [12]. Some methods $[13,15,17]$ focus on symbol timing. In [15], a method is proposed to distinguish the first significant multipath. Some methods for carrier frequency offset (CFO) estimation are proposed in $[14,16,18]$. The method in [16] is proposed based on the best linear unbiased estimation (BLUE) principle, which has a high performance. However, these methods all focus on optimizing the implementation complexity or performance of individual functions, and none of them offer optimization on the power consumption of a comprehensive synchronization process.

A comprehensive synchronization design for the MBOFDM UWB system is proposed in [9]. Based on autocorrelation (AC) algorithm, the design in [9] has low implementation complexity and can provide a comprehensive synchronization solution for the design of practical MB-OFDM UWB receiver. However, the major consideration of the design in [9] is implementation complexity. Low implementation complexity generally can bring a certain degree of power consumption decrease, but it is not equivalent to low power consumption. For example, the major consideration in a method for low implementation complexity is the hardware cost of the operation. However, in low-power design, not only the hardware cost of the operation but also the number of the operation to be executed need to be reduced.

With the development of very large scale integrated circuits (VLSI), the hardware cost of electronic products reduces greatly every year. Therefore, in recent years, low power consumption is usually more essential than low implementation complexity for electronic devices, especially the portable devices.

The design in this paper targets at providing a synchronization solution with very low power for the design of practical MB-OFDM UWB receivers. From the perspective of the comprehensive synchronization process, the power consumptions of the individual blocks in the proposed overall architecture are analyzed. Based on the result of the analysis, a novel TFC identification scheme with very low power and a novel packet detector with low power and high performance are proposed. In addition, a new matched filter with high noise immunity and low complexity is designed.

Using the same hardware implementation techniques, the algorithmic approach with lower computational complexity usually has lower power consumption. Therefore, in this paper, power consumption majorly refers to computational complexity and a method with low power refers to that the method has low computational complexity, as the major concern of the design is not the detailed circuits but the algorithmic methods.

The key contributions of this paper include the following:

- The analysis on the power consumption of the individual blocks from a new perspective. The power consumption of the individual blocks is analyzed from the perspective of the comprehensive synchronization process.

- A TFC identification scheme. In the scheme, a signal detector (SD) is designed to judge whether the preamble symbols are present, and a TFC type detector (TTD) is designed to identify TFC type. The TFC identification scheme has very low power consumption with satisfactory performance.

- A packet detector. In the packet detector, a coarse detector $(\mathrm{CD})$ is designed to detect symbols with low computational complexity and a final detector (FD) is designed to achieve high performance. The packet detector has low power with high performance.

- A matched filter. The matched filter has low computational complexity with high noise immunity. Moreover, the matched filter can roughly maintain the orthogonality between the preamble patterns and can be used to identify the preamble pattern.

- Different operations are implemented according to whether the preamble symbols are present or not. In this way, the power consumption of the blocks in the interval that no preamble symbol is present can be greatly reduced.

The rest of this paper is organized as follows. Section 2 gives a brief description of the signal and channel models. In Section 3, the power consumption of the scheme is analyzed. The individual blocks are designed in Section 4. Evaluation and simulation results are shown in Section 5. Section 6 concludes the paper.

\section{Signal and channel models}

The synchronization in the MB-OFDM UWB system is data-aided [2]. Dedicated preambles are used for synchronization. A standard preamble sequence consists of 21 packet synchronization (PS) symbols, 3 frame synchronization (FS) symbols, and 6 channel estimation (CE) symbols. In addition, zero-padded prefix intervals are used instead of cyclic prefix intervals in the symbol. A transmitted symbol is constructed by appending 32 null prefix samples, called zero-padded guard intervals, and five null guard samples to $N=128$ length IFFT output samples. A total of $N_{s}=165$ samples are included in a symbol. 
The IEEE 802.15.3a Task Group adopted a modified $\mathrm{S}-\mathrm{V}$ model for the UWB channel. The model is cluster based with independent fading and log-normal distribution of rays. The channel impulse response (CIR) can be expressed as

$$
h(t)=X \sum_{l=0}^{L} \sum_{k=0}^{K} a_{k, l} \delta\left(t-T_{l}-\tau_{k, l}\right),
$$

where $X$ is log-normal shading, $T_{l}$ is the delay of the $l$ th cluster, $\tau_{k, l}$ is the $k$ th ray delay related to the $l$ th cluster, and $a_{k, l}$ is multipath gain coefficient.

Let $s_{n}$ is the transmitted signal at the $n$th time index. The received signal $r_{n}$ can be expressed as

$$
r_{n}=\sum_{i=0}^{l-1} s_{n-i-\theta} h(i)+v_{n}
$$

where $l$ is the length of channel impulse response, $n$ is the time index, $\theta$ is the timing offset and $v_{n}$ is white Gaussian noise with zero mean and variance $\sigma^{2}$.

\section{Power consumption analysis}

To ensure that the designed individual blocks are compatible with each other and can construct a comprehensive synchronization solution for the design of practical MBOFDM UWB receiver, an overall architecture is proposed in this section. Also, the overall architecture is needed for analyzing the power consumptions of the synchronization blocks in the comprehensive synchronization process because the synchronization process is dependent on the overall architecture of the synchronizer. The block diagram of the proposed overall architecture is shown in Figure 1.

In MB-OFDM UWB systems, the data of the user are transmitted in the form of data block. One or several data frames are included in a data block. The structure of the data block is shown in Figure 2. In the figure, $A$ is the inter-block interval, $B$ is the inter-frame interval, $C$ is the time interval that used for packet detection and $D$ is the time interval used for symbol and frame timing. Let $N_{a}, N_{b}, N_{c}$, and $N_{d}$ are the numbers of symbols in $A, B, C$, and $D$, respectively. In most MB-OFDM UWB systems, $N_{a}$ (usually $>N$ ) is much larger than $N_{b}$ (usually $>40$ ), and $N_{b}$ is much larger than $N_{d}$ (usually <15) and $N_{c}$ (usually $<6$ ).

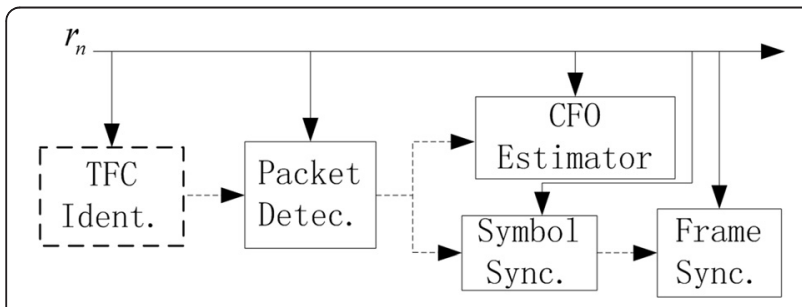

Figure 1 Block diagram of the proposed overall architecture.

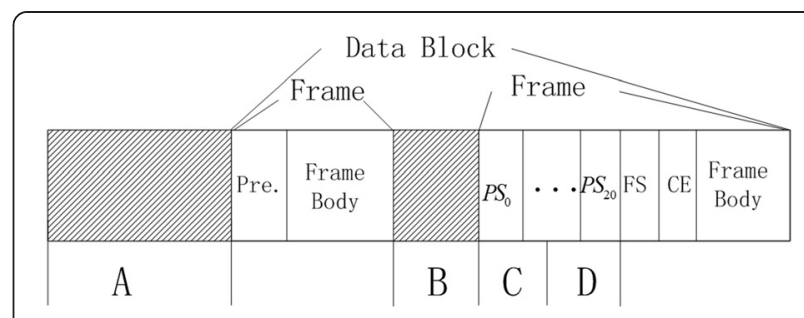

Figure 2 Structure of data block.

In Figure 1, the dashed line is the control line. It means that the individual function is activated by the condition that the previous individual function has been executed successfully. As shown from Figure 1, when signals are received, TFC identification is done firstly, and then the received signals go through the packet detection block. After packet detection is completed successfully, the timing offset and the CFO are estimated. Finally, frame synchronization is done.

Therefore, the TFC identification block needs to detect almost all of the symbols in $A$ because the position of the PS symbols is unknown to it. Another characteristic of TFC identification is that it does not need to be executed for each received frame. For example, because the TFC type has been identified during the first received frame, TFC identification is not executed any more when the second frame of the same data block is received. However, the packet detection block needs to be executed for each received frame to avoid offset accumulation. Therefore, almost all the symbols in $B$ need to be detected by the packet detection block. Thus, a total of about $N_{a} N_{s}$ and $N_{b} N_{s}$ times detections have been executed by the TFC identification block and the packet detection block, respectively, before PS symbols are present.

Let $n \hat{~ i s ~ t h e ~ t i m e ~ i n d e x ~ a t ~ w h i c h ~ p a c k e t ~ i s ~ d e t e c t e d . ~}$ Symbol timing is to estimate the time offset between $n \wedge$ and the exact starting boundary of the symbol. The time offset is not large (usually $<10$ samples). Therefore, only the samples, which are closed to $\hat{n}$, need to be detected for estimating the time offset. Because $N_{d}<15$, a total of less than $2 N$ times detections are executed in the whole process of symbol and frame timing offset estimation. For CFO estimation, the CFO estimation block does not need to wait the arrival of PS symbols because PS symbols have been detected by the packet detection block. According to the view in [3], the simplest CFO estimation method can be implemented with one complex multiplication.

Therefore, most of the samples that detected by the synchronizer are used for TFC identification and packet detection. Only optimizing the implementation complexity is not low-power solution for TFC identification and packet detection. However, because the number of 
samples used for CFO estimation and timing offset estimation is small, the normal method with low implementation complexity is low-power design. Therefore, the key for implementing the synchronizer with low power is designing the TFC identification block and the packet detection block with low power consumption.

\section{Individual functions}

\subsection{TFC identification}

In MB-OFDM UWB systems, the transmitted data are spread on the frequency bands of the same band group, and TFCs are used for frequency hopping. The TFCs in specification [1] are shown in Table 1.

During time interval $A$ (in Figure 2), the most concern of the TFC identification process is not identifying TFC type but judging whether the PS symbol is present or not.

In this paper, we design a SD to judge whether PS symbols are present. In MB-OFDM UWB systems, the repeated PS symbols are used for synchronization. The transmitted sample at time index $n+d N_{s}$ ( $d$ is the symbol interval on one frequency band of the used TFC type) is identical to the sample at the $n$th time index. Thus, at receiver, $\left|r_{n}+r_{n+d N_{s}}\right|$ is larger than $\left|r_{n}\right|$ and $\left|r_{n+d N_{s}}\right|$ with very high probability. However, if noise is transmitted at points $n$ and $n+d N_{s}, r_{n}$ and $r_{n+d N_{s}}$ have opposite polarity with high probability $(\approx 1 / 2)$. Therefore, $\sum_{m}\left|r_{n+m}+r_{n+d N_{s}+m}\right|$ can be used for detecting the repeated PS symbols.

As shown in Table 1 , if $d=6$, the transmitted data can be received again at the same frequency band for all TFC types. Let $W_{n}$ be the accumulated value of $\left|r_{n}+r_{n+6 N_{s}}\right|$ at the $n$th time index.

$$
W_{n}=\sum_{k=0}^{N-1}\left|r_{n+k}+r_{n+k+6 N_{s}}\right|
$$

By using iterative structure, $W_{n}$ can be rewritten as

$$
W_{n}=W_{n-1}+\left|r_{n}+r_{n+6 N_{s}}\right|-\left|r_{n-N}+r_{n+6 N_{s}-N}\right|
$$

In this way, the major operations for calculating $W_{n}$ are three real additions. The structure of the SD is shown in Figure 3b.

Table 1 The TFCs in specification [1]

\begin{tabular}{llllllll}
\hline TFC number & Preamble pattern & \multicolumn{7}{l}{ Time-frequency code } \\
\hline 1 & 1 & 1 & 2 & 3 & 1 & 2 & 3 \\
2 & 2 & 1 & 3 & 2 & 1 & 3 & 2 \\
3 & 3 & 1 & 1 & 2 & 2 & 3 & 3 \\
4 & 4 & 1 & 1 & 3 & 3 & 2 & 2 \\
5 & 1 & 1 & 2 & 1 & 2 & 1 & 2 \\
6 & 2 & 1 & 1 & 1 & 2 & 2 & 2 \\
\hline
\end{tabular}

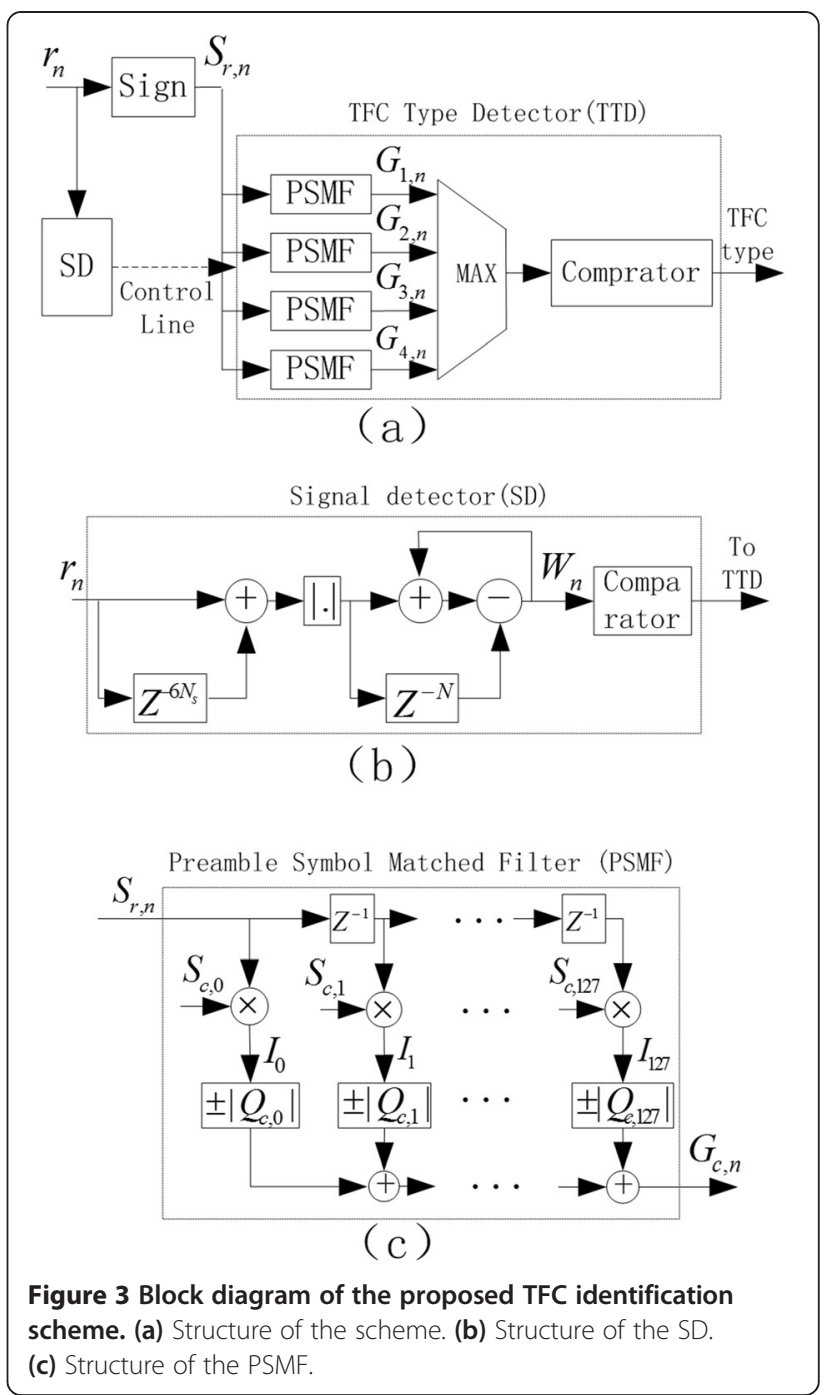

Once the SD determines that PS symbols are present, a TTD is activated to identify the used TFC type. The CC operation is not sensitive to noise and is suitable for TFC identification in low signal-to-noise ratios (SNRs). However, the computational complexity of CC operation is very high. For example, if it is directly used to identify TFC type in a MB-OFDM UWB system of specification [1], $4 N$ complex multiplications need to be executed when a sample is received.

In this paper, the proposed TTD is shown in Figure 3a. To simplify the complexity of CC algorithm, a PSMF is designed. The structure of the PSMF is shown in Figure 3c. In Figure 3c, $S_{r, n}$ is the sign-bit of the received sample at the $n$th time index, $S_{c, k}$ is the sign-bit of the $k$ th coefficient of preamble pattern $c(c=1,2,3,4), Q_{c, k}$ is an integer and is the rounding of the $k$ th coefficient of preamble pattern $c$, and $G_{c, n}$ is the output of the PSMF at the $n$th 
time index. First, $S_{r, n}+k$ compares with $S_{c, k}$, and the comparison result $I_{n+k}$ is given by

$$
I_{n+k}=\left\{\begin{array}{cr}
1 & S_{r, n+k}=S_{c, k} \\
-1 & S_{r, n+k}=-S_{c, k}
\end{array}\right.
$$

After $I_{n+k}(k=0,1, \ldots N-1)$ are achieved,

$$
G_{c, n}=\sum_{k=0}^{N-1} I_{n+k}\left|Q_{c, k}\right|
$$

Of the coefficients in the preamble patterns in specification [1], some have large absolute value $(\approx 3)$, and some have the small absolute value $(\approx 0)$. By assuming $c=i$ and $G_{i, n}$ is the output of the PSMF at the $n$th time index, there are three scenarios of the transmitted samples:

(1) The coefficients of preamble pattern $i$ are transmitted. In this case, the mean probability of $I_{n+k}(k=0,1, \ldots$ $N-1)=1$ is much higher than that of $I_{n+k}(k=0,1, \ldots$ $N-1)=-1$. Moreover, the signs of the coefficients with larger absolute value are more unlikely be changed at the receiver. Therefore, most of $I_{n+k}=-1$ occurs on the coefficients with small absolute value. In this case, $G_{i, n}$ is close to $\sum_{k=0}^{N-1}\left|Q_{i, k}\right|$.

(2) The noise is transmitted. In this case, the probability of $I_{n+k}=1$ is $1 / 2$. Therefore, for most $G_{i, n}$ in this case, $G_{i, n} \approx 0$.

(3) The coefficients of preamble pattern $j(j \neq i)$ are transmitted. In this case, the mean probability of $I_{n+k}(k=0,1, \ldots N-1)=1$ is approximately $1 / 2$ because the orthogonality between the preamble patterns. In addition, the occurrence of $I_{n+k}=1$ are uncorrelated and independent with the absolute value of the coefficient. Therefore, for most $G_{i, n}$ in this case, $G_{i, n} \approx 0$.

Therefore, the preamble pattern can be identified by comparing the maximum of $\left\{G_{c, n} \mid c=1,2,3,4\right\}$ with a predefined threshold. If $G_{i, n}=\max \left\{G_{c, n} \mid c=1,2,3,4\right\}$ and $G_{i, n}$ is larger than the threshold, preamble pattern $i$ is determined as the preamble pattern used in transmission.

After the preamble pattern has been identified, TFC type can be identified easily. As seen in Table 1, TFC 1 and TFC 2 share the same preamble pattern with TFC 5 and TFC 6, respectively. Of TFC 3 and TFC 4, each occupies one preamble pattern exclusively. Therefore, if TFC 3 or TFC 4 is used, the TFC type can be directly identified by identifying preamble pattern. If TFC $1,2,5$, or 6 is used, the TFC type can also be identified easily in an additional step according to the hopping order of the TFC type. For example, we assume that preamble pattern 1 is determined as the right preamble pattern at the $n$th time index. If TFC 5 is used, the signal would be received again at $G_{1, n+2 N_{s}}$ on the same frequency band. For TFC 1 , the point is $G_{1, n+3 N_{s}}$. Therefore, we can compare $G_{1, n+2 N_{s}}$ with $G_{1, n+3 N_{s}}$. If $G_{1, n+2 N_{s}}>G_{1, n+3 N_{s}}$, TFC 5 is the TFC type used in transmission, else TFC 1 is used.

\subsection{Packet detection}

As analyzed in Section 3, for the methods which employ the same operations regardless of whether PS symbols are present or not, most of the power consumption during the packet detection process is cost in interval $B$. Moreover, because errors occur in the TFC identification process can be corrected easily by the following packet detection process but errors occur in the packet detection process can hardly be corrected by the following processes such as symbol timing, the performance requirement for packet detection is much higher than that for TFC identification.

In this paper, a packet detector, which divides the packet detection process into two steps, is proposed. The first step is to detect symbols with low computational complexity, and the second step targets at obtaining high performance.

The major components of the proposed packet detection scheme are a CD and a FD. The CD is designed to detect the received signals with low computational complexity and low miss detection probability by allowing high false alarm probability. The FD is designed to confirm the PS symbols detected by the CD with both low miss detection probability and low false alarm probability.

The structure of the $C D$ is shown in Figure 4b. In the $\mathrm{CD}$, a CC block, which based on the sign-bit CC algorithm $[14,15]$, is designed. In the CC block, only the even samples are used for CC operation. The outputs of the CC block are given by

$$
E_{n}=\sum_{k=0}^{1 / 2 N-1} S_{r, n+2 k} S_{c, 2 k}^{*}
$$

where $S_{r, n+2 k}$ is the sign of the received sample at time index $n+2 k$ and $S_{c, 2 k}$ are the sign of the $2 k$ th coefficient of preamble pattern $c$.

However, the noise immunity of the $\mathrm{CC}$ will be greatly deteriorated by shorting the length to N/2. Though low false alarm probability is not required for the $\mathrm{CD}$, too high false alarm probability will decrease the effectiveness of it. To improve the noise immunity of the $C D$, the CC output with $d N_{s}$ delay can be added to $E_{n}$, where $d N_{s}$ is the inter-symbol spacing on a frequency band. In Figure $4 \mathrm{~b}, U_{n}$ is given by

$$
U_{n}=E_{n}+E_{n+d N_{S}}
$$

When different TFC type is used, $d$ is different. As seen from Table 1 , 

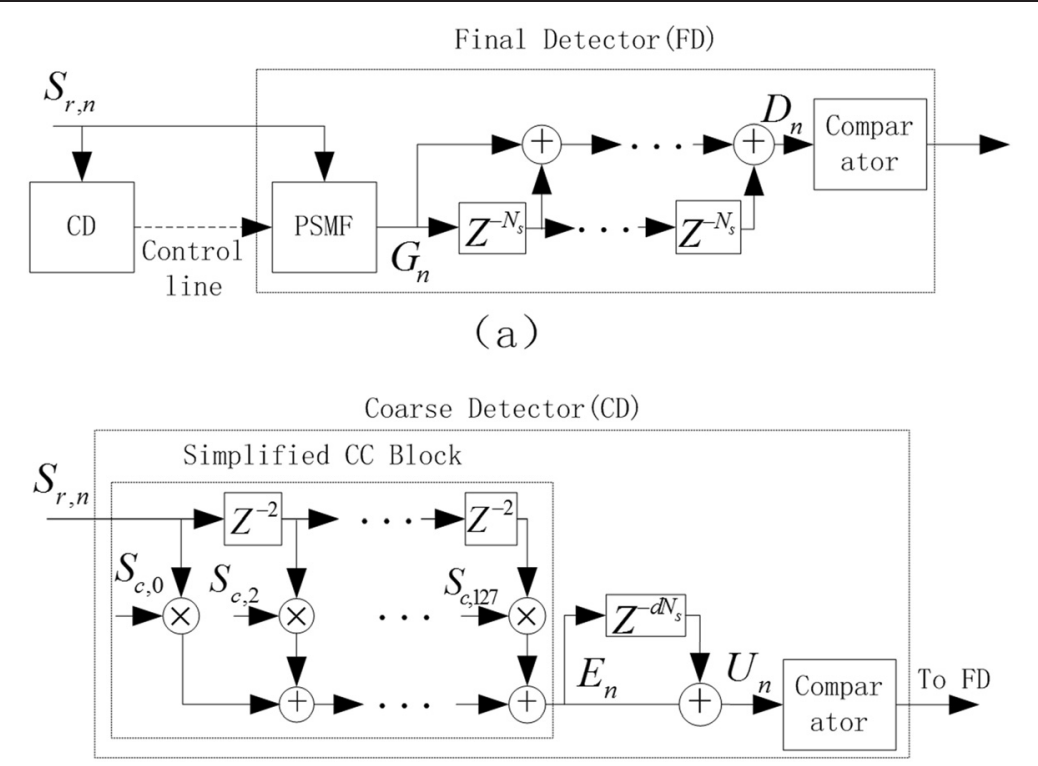

(b)

Figure 4 Block diagram of the proposed packet detector. (a) Structure of the packet detector. (b) Structure of the CD.

$$
d=\left\{\begin{array}{cc}
3 & \text { if TFC } 1 \text { or } 2 \text { is used } \\
2 & \text { if TFC } 5 \text { is used } \\
1 & \text { if TFC } 3,4, \text { or } 6 \text { is used }
\end{array}\right.
$$

In the $\mathrm{CD}, U_{n}$ is compared with a predefined threshold. If $U_{n}$ is larger than the threshold, the PS symbol is detected by the CD.

However, because the threshold for the $\mathrm{CD}$ is selected with very small value to ensure low miss detection probability, the false alarm probability of the $\mathrm{CD}$ is still much high. This means that many PS symbols detected by the $\mathrm{CD}$ are not real PS symbols. The FD is designed to confirm the results of the CD. The structure of the FD is shown in Figure 4a. The FD is designed based on the PSMF, which is designed in the TFC identification scheme.

Because the accumulated value of several outputs of the PSMF has better noise immunity, several outputs of the PSMF are accumulated to compare with a predefined threshold. In Figure $4 \mathrm{a}, D_{n}$ is the accumulated value. As TFC type has been identified and the CD has detected a PS symbol, frequency hopping can be used in the FD. Therefore, $D_{n}$ is given by

$$
D_{n}=\sum_{i=0}^{M} G_{n+i N_{s}}
$$

where $G_{n+i N_{s}}$ is the output of the PSMF at time index $n+i N_{s}(i=0,1, \ldots, M), N_{s}$ is the length of a symbol (also the sample interval between two consecutive symbols) and $M$ is the number of symbols used by the FD ( $M$ can be selected according to the performance requirement of the actual system). If $D_{n}$ is larger than the predefined threshold for the $\mathrm{FD}$, packet is detected.

\section{Evaluation}

The proposed design is evaluated in this section. The computational complexities of the proposed methods are evaluated. Also, the performances of them are simulated. The parameters used in simulation follow ones' in specification [1]: for $N=128, N_{s}=165$, the three carrier frequencies are $\{3432,3960,4488\} \mathrm{MHz}$ and the sub-carrier spacing $f_{i}=4.125 \mathrm{MHz}$.

\subsection{TFC identification}

The performance of the SD is first estimated. The simulated miss detection probabilities of the SD under different false alarm probabilities are shown in Figure 5. In the simulation, TFC 1 and CM1 are used. To achieve the given false alarm probability, the threshold for each SNR is optimized by searching the suitable value. The miss detection probability of the SD is simulated under the condition that the given false alarm probability is $0.2,0.45$, and 0.7 , respectively. When the given false alarm probability is 0.45 , the miss detection probabilities are approximately 0.09 and 0.008 with $\mathrm{SNR}=-8 \mathrm{~dB}$ and $\mathrm{SNR}=-5 \mathrm{~dB}$, respectively, which are satisfactory values for most systems. In addition, the computational complexity of the SD is about 1/16 of that of the TTD (analyzed in the last paragraph of this subsection). Therefore, SD is effective for reducing the computational complexity. 

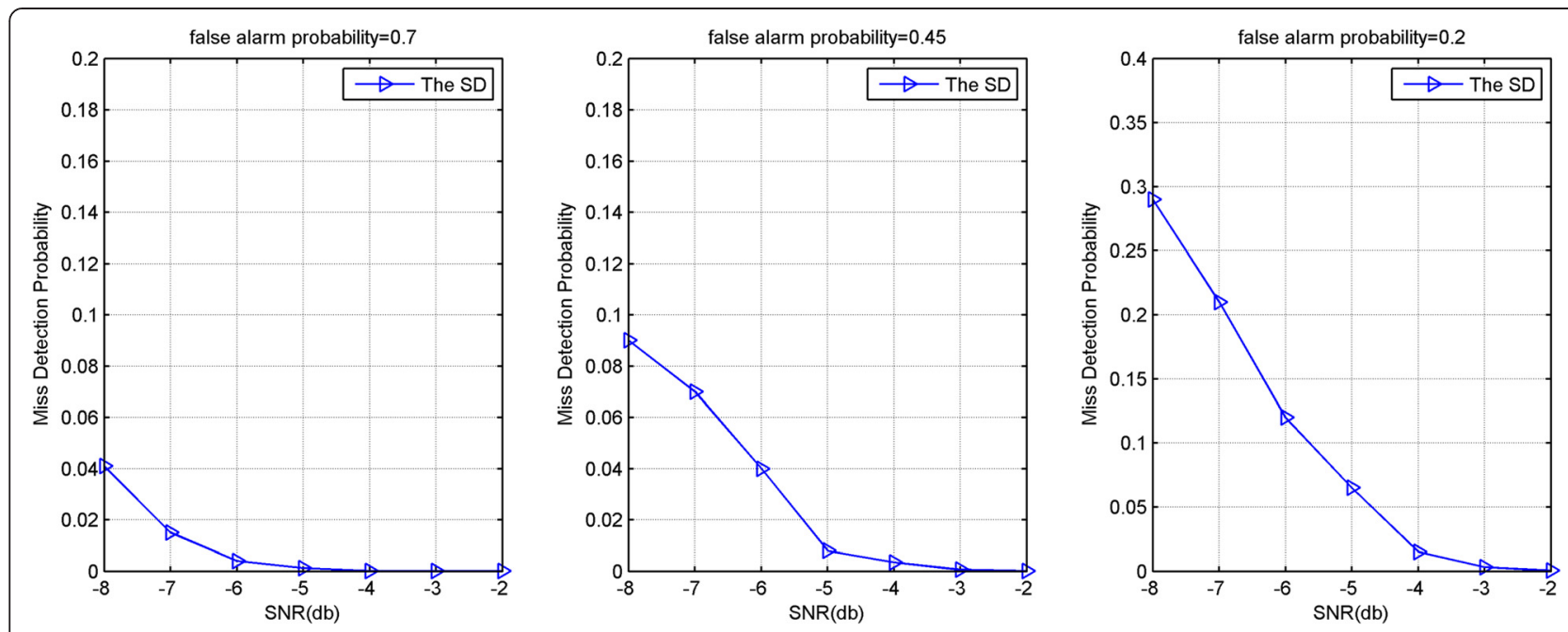

Figure 5 The miss detection probabilities of the SD. Under TFC 1 and the CM1 channel, the false alarm probability of 0.2 , 0.45 , and 0.7 is assumed, respectively.

The performance of the TTD is also evaluated. If the PSMF for preamble pattern $i$ is considered, there are three scenarios of the transmitted samples:

(1) The transmitted samples are the coefficients of preamble pattern $i$. In this case, let $P_{1, \lambda}=P\left[G_{i, n}>\lambda\right]$. According to (26) in Appendix 1,

$$
P_{1, \lambda} \approx 1-\Phi\left(\frac{\frac{N+2 \lambda}{4}-\frac{1}{2} \operatorname{erfc}\left(\frac{-\bar{m}}{\sqrt{2} \sigma^{2}}\right) N}{\sqrt{\frac{1}{2} \operatorname{erfc}\left(\frac{-\bar{m}}{\sqrt{2} \sigma^{2}}\right)\left[1-\frac{1}{2} \operatorname{erfc}\left(\frac{-\bar{m}}{\sqrt{2} \sigma^{2}}\right)\right] N}}\right),
$$

where $\operatorname{erfc}()$ is the complementary error function, $\bar{m}$ is the mean of the absolute values of the preamble coefficients and $\Phi(x)=\int_{-\infty}^{x} \frac{1}{\sqrt{2 \pi}} e^{-\frac{1}{2} t^{2}} d t$.

(2) The transmitted samples are noise. In this case, let $P_{2, \lambda}=P\left[G_{i, n}>\lambda\right]$. According to (29) in Appendix 1,

$$
P_{2, \lambda} \approx 1-\Phi\left(\frac{\frac{N+\lambda}{2}-\frac{1}{2} N}{\frac{1}{2} \sqrt{N}}\right)
$$

(3) The transmitted samples are the coefficients of preamble pattern $j(j \neq i)$. In this case, let $P_{3, \lambda}=P\left[G_{i, n}>\lambda\right]$. According to (30) in Appendix 1,

$$
P_{3, \lambda} \approx 1-\Phi\left(\frac{\frac{N+\lambda}{2}-\frac{1}{2} N}{\frac{1}{2} \sqrt{N}}\right)
$$

Let $P_{t e e}$ be the error probability of the TTD and $\lambda$ is the predefined threshold for the TTD. If TFC 3 or 4 is used in the transmission, the TFC type can be identified directly by identifying the preamble pattern. In the case that TFC 3 or 4 is used, $P_{t_{-} e}$ can be given by

$$
P_{t_{-} e} \approx\left(1-P_{1, \lambda}\right)+4 P_{2, \lambda}
$$

If TFC $1,2,5$, or 6 is used, an additional step is needed to identify the TFC after the preamble pattern has been identified. In this case, $P_{t_{-} e}$ is given by

$$
\begin{aligned}
& P_{t_{-} e} \approx\left(1-P_{1, \lambda}\right)+4 P_{2, \lambda}+2\left(1-P_{1, \lambda}\right) P_{2, \lambda} \\
& \approx\left(1-P_{1, \lambda}\right)+4 P_{2, \lambda}
\end{aligned}
$$

As to the overall performance of the proposed TFC identification scheme, the overall performance would be deteriorated by taking the miss detection of the SD into consideration, but it would be improved by taking the false alarm of the SD into consideration. By selecting an appropriate false alarm probability for the SD, the influence of the SD to the overall performance can be ignored. Therefore, $P_{t_{-} e}$ can be seen as the error probability of the proposed TFC identification scheme.

Figure 6 shows the error probability comparison among TFC identification methods. The scheme in [9] and the traditional CC algorithm are included in the comparison. In the simulation, TFC 1 and the CM1 channel are used. The predefined thresholds used in the methods are all optimized by searching the optimal value for each SNR. As shown in Figure 6, the traditional CC algorithm has the best performance, and the performance of the proposed scheme is close to that of the traditional CC algorithm. In actuality, the performance of the traditional CC algorithm is optimal among the TFC identification algorithms. However, the complexity of it is too high to be used in most actual systems. 


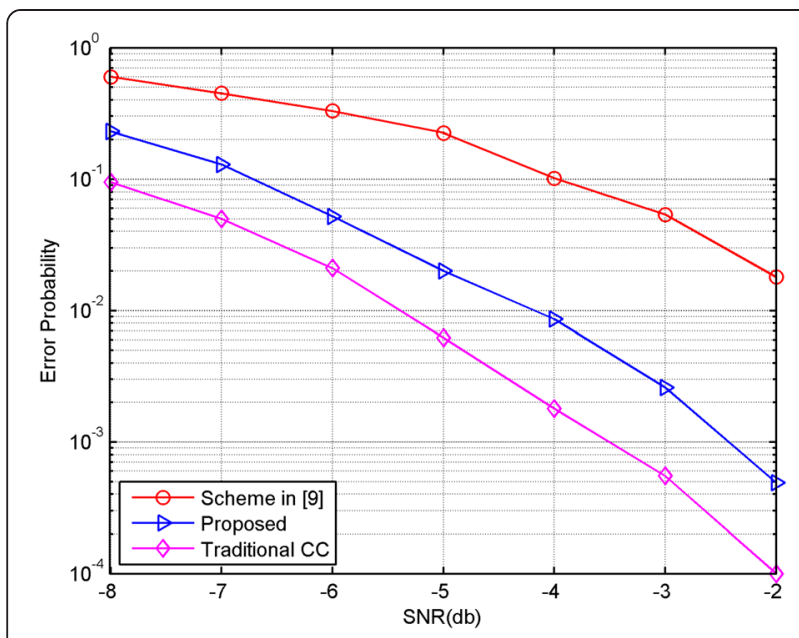

Figure 6 Error probabilities of TFC identification methods under TFC 1 and the CM1 channel.

The comparison on computational complexity among TFC identification methods is shown in Table 2. The scheme in [9] and the traditional CC algorithm are included in the comparison. In the table, $D_{a}$ is the number of the symbols in interval $A$. In the proposed scheme, the adders of the PSMF in the TTD can be implemented by 64 3-, 32 4-, 16 5-, and 8 9-bit integer adders. The computational complexity of these adders is approximately equal to that of eight real adders. Thus, 128 XNOR gates and eight real adders can be seen as the major components of the PSMF. In the proposed scheme, we assume that the false alarm of SD is 0.45 . Therefore, to complete a TFC identification process, about $3 N_{s} D_{a}$ real additions, $N_{s} D_{a}$ real comparisons, $N_{s} D_{a}$ taking absolute value operations (ABS), and $N_{s} D_{a}$ XNOR gate operations are consumed by the $\mathrm{SD}$, and about $4 \times 8 \times 0.45 N_{s} D_{a}$ real additions, $4 \times 0.45 N_{s} D_{a}$ integer comparison, and $4 \times$ $128 \times 0.45 N_{s} D_{a} \mathrm{XNOR}$ gate operations are consumed by the TTD. To simplify the comparison in Table 2, the computational complexity of three integer additions is equal to that of one real addition, three integer comparisons are converted to one real comparison, and eight $\mathrm{XNOR}$ gates are converted to one integer comparator. In Table 2, the computational complexities of the real addition and the real comparison are far lower than that

Table 2 Comparison on computational complexity among TFC identification methods

\begin{tabular}{llll}
\hline Operation & Proposed & Scheme in [9] & CC algorithm \\
\hline $\begin{array}{l}\text { Complex } \\
\text { multiplication }\end{array}$ & 0 & $4 N_{s} D_{a}$ & $4 \times 128 N_{s} D_{a}$ \\
Real addition & $(3+14.4) N_{s} D_{a}$ & $8 N_{s} D_{a}$ & $4 \times 127 N_{s} D_{a}$ \\
Real comparison & $(1.04+10.2) N_{s} D_{a}$ & $4 N_{s} D_{a}$ & $4 \times N_{s} D_{a}$ \\
ABS & $N_{s} D_{a}$ & $4 N_{s} D_{a}$ & $4 \times 128 N_{s} D_{a}$ \\
\hline
\end{tabular}

of the complex multiplication but are much higher than that of the ABS. As the complex multiplication is not used, the proposed scheme has much lower computational complexity than the other methods in the table.

\subsection{Packet detection}

The performance of the CD is evaluated firstly. Let $P_{c \_m}$ be the miss detection probability of the CD. According to (35) in Appendix 2, $P_{c \_m}$ is given by

$$
P_{c \_m} \approx \Phi\left(\frac{\frac{N+\lambda_{c}}{2}-\frac{N}{2} \operatorname{erfc}\left(\frac{-\bar{a}}{\sqrt{2} \sigma^{2}}\right)}{\sqrt{\frac{N}{2} \operatorname{erfc}\left(\frac{-\bar{a}}{\sqrt{2} \sigma^{2}}\right)\left[1-\frac{1}{2} \operatorname{erfc}\left(\frac{-\bar{a}}{\sqrt{2} \sigma^{2}}\right)\right]}}\right)
$$

where $\bar{a}$ is the mean of the absolute values of the even coefficients and $\lambda_{c}$ is the threshold for the CD.

Let $P_{c_{-} f}$ be the false alarm probability of the CD. According to (36) in Appendix 2, $P_{c_{-} f}$ is given by

$$
P_{c_{-} f} \approx 1-\Phi\left(\frac{\frac{N+\lambda_{c}}{2}-\frac{1}{2} N}{\frac{1}{2} \sqrt{N}}\right)
$$

The simulated miss detection probabilities of the CD under different false alarm probabilities are shown in Figure 7. In the simulation, TFC 1 and the CM1 channel are used. The simulated results are achieved by assuming the false alarm probability is $0.1,0.2$, and 0.3 , respectively. If 0.2 is the given false alarm probability, the miss detection probabilities are lower than 0.008 and 0.001 with $\mathrm{SNR}=-8 \mathrm{~dB}$ and $\mathrm{SNR}=-6 \mathrm{~dB}$, respectively. These miss detection results are low enough to satisfy most MB-OFDM systems, and the computational complexity of the CD is approximately $1 / 3$ of that of the FD. So, the proposed CD is effective for reducing computational complexity.

The performance of the FD is also evaluated. Let $P_{f_{-} m}$ be the miss detection probability of the FD and $\lambda_{f}$ is the threshold for the FD. According to (38) in Appendix 2, $P_{f_{-} m}$ is given by

$$
P_{f \_m} \approx \Phi\left(\frac{\frac{N+\lambda_{f}}{2}-\operatorname{erfc}\left(\frac{-\bar{m}}{\sqrt{2} \sigma^{2}}\right) N}{\sqrt{\operatorname{erfc}\left(\frac{-\bar{m}}{\sqrt{2} \sigma^{2}}\right)\left[1-\frac{1}{2} \operatorname{erfc}\left(\frac{-\bar{m}}{\sqrt{2} \sigma^{2}}\right)\right] N}}\right)
$$

Let $P_{f_{-} f}$ be the probability of false alarm of the FD. According to (39) in Appendix 2, $P_{f_{-} f}$ is given by

$$
P_{f_{-} f} \approx 1-\Phi\left(\frac{\frac{2 N+\lambda_{f}}{2}-N}{\frac{1}{2} \sqrt{2 N}}\right)
$$

Let $P_{p_{-} e}$ be the error probability of the proposed packet detection scheme. $P_{p_{-} e}$ is given by 

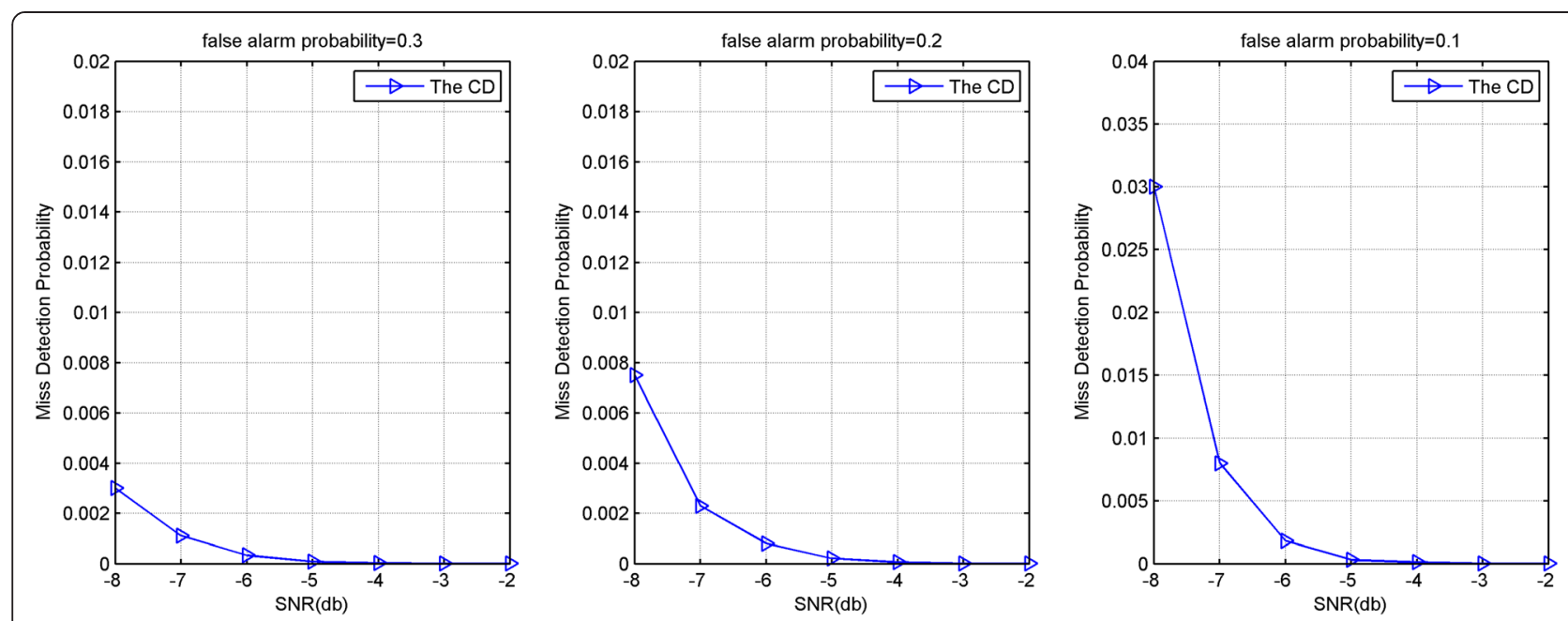

Figure 7 Miss detection probabilities of the CD. Under TFC 1 and the CM1 channel, the false alarm probability of $0.1,0.2$, and 0.3 is assumed, respectively.

$$
P_{p_{-}}=P_{c \_m}+P_{f \_m}+P_{c \_f} P_{f-f}
$$

By selecting an appropriate false alarm probability for the $\mathrm{CD}$, the influence of the $\mathrm{CD}$ on $P_{p_{-} e}$ can be ignored. Therefore, $P_{p_{-} e}$ can be simplified as

$$
P_{p_{-} e}=P_{f_{-} m}+P_{f_{-} f}
$$

The simulated error probabilities of packet detection methods are shown in Figures 8 and 9. Except the proposed packet detector, the error probabilities of the traditional AC algorithm, the traditional CC algorithm, and the sign-bit CC-based method in [12] are also shown in the figures. In the simulation, two channel types, CM1 and CM4, and TFC 1 are used, and the threshold of each method is optimized by searching the optimal value for

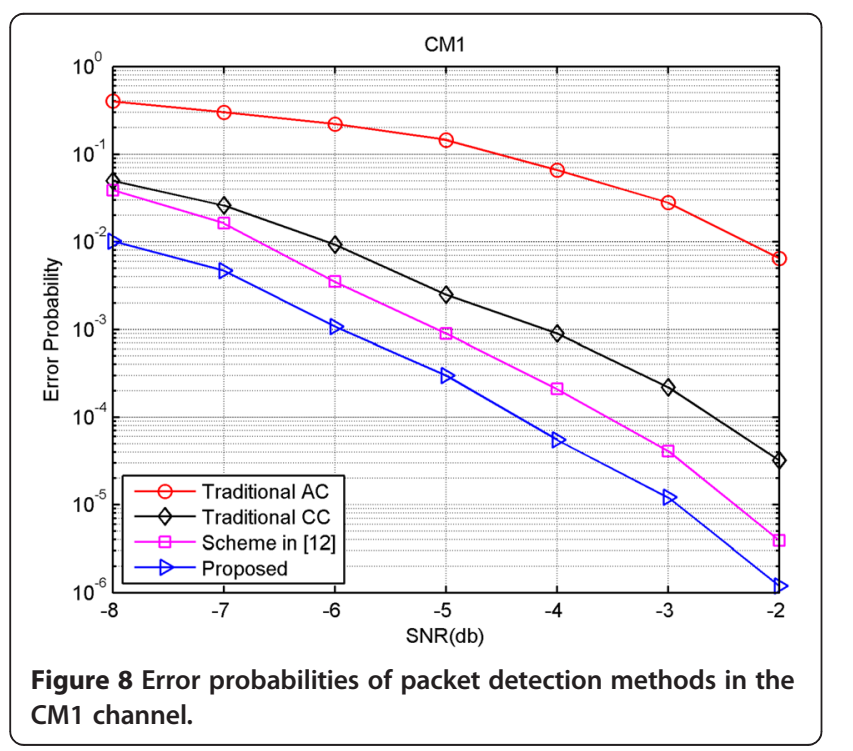

each SNR. As seen from Figures 8 and 9, the proposed method has the lowest error probability among the methods in the figures.

The comparison on computational complexity among packet detectors is shown in Table 3. The traditional AC algorithm, the traditional CC algorithm, and the method in [12] are included in the comparison. In Table $3, D_{b}$ is the number of the symbols in interval $B$. We assume the false alarm of the CD is 0.2 . The CC block in the $C D$ can be implemented with 64 XNOR gates and the adders of 32 2-, 16 3-, 8 4-, and 4 7-bit integer adders. The computational complexity of these adders is about equal to that of three real adders. In the packet detection process, the $\mathrm{CD}$ approximately consumes $3 N_{s} D_{b}$ real additions, $64 N_{s} D_{b}$ XNOR gate operations, and $N_{s} D_{b}$

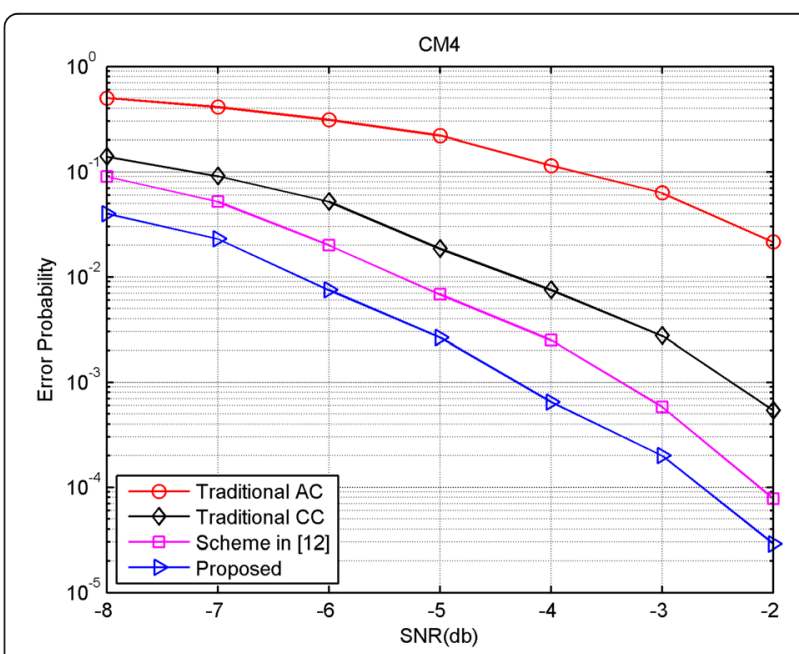

Figure 9 Error probabilities of packet detection methods in the CM4 channel. 
Table 3 Comparison on computational complexity among packet detectors

\begin{tabular}{lllll}
\hline Operation & Proposed & Scheme in [12] & Traditional AC & Traditional CC \\
\hline Complex multiplication & 0 & $1 / 10 N_{s} D_{b}$ & $N_{s} D_{b}$ & $128 N_{s} D_{b}$ \\
Real addition & $(3+1.6) N_{s} D_{b}$ & $6 N_{s} D_{b}$ & $2 N_{s} D_{b}$ & $127 N_{s} D_{b}$ \\
Real comparison & $(3+1.1) N_{s} D_{b}$ & $5.6 N_{s} D_{b}$ & $N_{s} D_{b}$ & $N_{s} D_{b}$ \\
ABS & 0 & $N_{s} D_{b}$ & $N_{s} D_{b}$ & $128 N_{s} D_{b}$ \\
\hline
\end{tabular}

integer comparisons, and the FD approximately needs $0.2 \times 8 N_{s} D_{b}$ real additions, $0.2 N_{s} D_{b}$ integer comparisons, and $0.2 \times 128 N_{s} D_{b}$ XNOR gate operations. To simplify the comparison, the computational complexity of the adders of the CC block in [12] is equal to that of six real adders, and the computational complexity of the integer multipliers in [12] is equal to $1 / 10$ the computational complexity of one complex multiplier. As seen from Table 3, the proposed scheme has much lower computational complexity than the other schemes.

\section{Conclusions}

In this paper, a power-efficient synchronization design is proposed for MB-OFDM UWB systems. Based on the proposed overall architecture, the power consumptions of the individual blocks are analyzed from the perspective of the comprehensive synchronization process. A TFC identification structure with very low power consumption is proposed. A structure with low computational complexity and high performance is proposed for packet detection. In addition, a matched filter, which has low computational complexity and can be used for identifying TFC type, is designed.

\section{Appendix 1 The probabilities of $G_{c, n}>\lambda$}

If we assume $c=i$ in Equation 6, then the PSMF for preamble pattern $i$ is considered and $G_{i, n}$ is the output of the PSMF at the $n$th time index. The probabilities of $G_{i, n}>\lambda$ of the three scenarios of the transmitted samples are analyzed as follows:

(1) The transmitted samples are the coefficients of preamble pattern $i$. In this case, if $s_{n}+k$ is transmitted and is received as $r_{n+k}$ at the receiver, $r_{n+k}$ follows a Gaussian distribution with mean $s_{n+k}$. In (6), the value domain of $\left|Q_{c, k}\right|(k=0,1, \ldots$, $N-1)$ is $\{0,1,2,3\}$. Because most $I_{n+k}=-1$

occur on the coefficients with small absolute value, the mean of $I_{n+k}\left|Q_{c, k}\right|\left(k=0,1, \ldots, N-1 ; I_{n+k}=-\right.$ 1 ) is approximately equal to $-1 / 2$. Also, usually $I_{n+k}=1$ occurs on the coefficients with large absolute value. The mean of $I_{n+k}\left|Q_{c, k}\right|(k=0,1, \ldots$, $N-1 ; I_{n+k}=1$ ) is approximately equal to $3 / 2$. To simplify the analysis, $I_{n+k}\left|Q_{c, k}\right|$ is simplified as

$$
I_{n+k}\left|Q_{c, k}\right|=\left\{\begin{array}{lr}
-1 / 2 & I_{n+k}=-1 \\
3 / 2 & I_{n+k}=1
\end{array}\right.
$$

In this case, if the sign of $r_{n+k}$ is equal to that of $s_{n+k}$, $I_{n+k}=1$. Let $p_{k}$ be the probability of $I_{n+k}=1$, and $\bar{p}$ is the mean of $p_{k}(k=0,1, \ldots N-1) \cdot \bar{p}$ can be given by

$$
\bar{p} \approx \frac{1}{2} \operatorname{erfc}\left(\frac{-\bar{m}}{\sqrt{2} \sigma^{2}}\right)
$$

where $\operatorname{erfc}()$ is the complementary error function, and $\bar{m}$ is the mean of the absolute values of the preamble coefficients. Because $N(=128)$ is large, according to the central limit theorem, $P_{1, \lambda}$ is given by

$$
\begin{aligned}
& P_{1, \lambda} \approx \frac{1}{\sqrt{2 \pi}} \int_{\left(\frac{N+2 \lambda}{4}-N p\right) / \sqrt{N p(1-p)}}^{\infty} e^{-\frac{1}{2} t^{2}} d t \\
& =1-\Phi\left(\frac{\frac{N+2 \lambda}{4}-\frac{1}{2} \operatorname{erfc}\left(\frac{-\bar{m}}{\sqrt{2} \sigma^{2}}\right) N}{\sqrt{\frac{1}{2} \operatorname{erfc}\left(\frac{-\bar{m}}{\sqrt{2} \sigma^{2}}\right)\left[1-\frac{1}{2} \operatorname{erfc}\left(\frac{-\bar{m}}{\sqrt{2} \sigma^{2}}\right)\right] N}}\right)
\end{aligned}
$$

where $(x)=\int_{-\infty}^{x} \frac{1}{\sqrt{2 \pi}} e^{-\frac{1}{2} t^{2}} d t$. In (25), not $\lambda$ but $\frac{N+2 \lambda}{4}$ is used because not $\{0,1\}$ of the Bernoulli distribution but $\{-1 / 2,3 / 2\}$ is the value domain for $I_{n+k}\left|Q_{c, k}\right|(k=0,1$, .., $N-1)$.

(2) The transmitted samples are noise. In this case, the received samples are Gaussian noise with zero mean. In this case, to simplify the analysis, $I_{n+k}\left|Q_{c, k}\right|$ can be simplified as

$$
I_{n+k}\left|Q_{c, k}\right|=\left\{\begin{array}{cr}
-1 & I_{n+k}=-1 \\
1 & I_{n+k}=1
\end{array}\right.
$$

In this case, the probability of $I_{n+k}=1$ is equal to $\frac{1}{2}$. $P_{2, \lambda}$ is given by

$$
\begin{aligned}
& P_{2, \lambda} \approx \frac{1}{\sqrt{2 \pi}} \int_{\left(\frac{N+\lambda}{2}-N p\right) / \sqrt{N p}(1-p)}^{\infty} e^{-\frac{1}{2} t^{2}} d t \\
& =1-\Phi\left(\frac{\frac{N+\lambda}{2}-\frac{1}{2} N}{\frac{1}{2} \sqrt{N}}\right)
\end{aligned}
$$

(3) The transmitted samples are coefficients of preamble pattern $j(j \neq i)$. Because the coefficients of preamble pattern $j(j \neq i)$ are orthogonal with the 
coefficients of preamble pattern $i$, the received samples can be seen as noise for calculating $G_{i, n}$. Therefore, $P_{3, \lambda}$ is given by

$$
P_{3, \lambda} \approx 1-\Phi\left(\frac{\frac{N+\lambda}{2}-\frac{1}{2} N}{\frac{1}{2} \sqrt{N}}\right)
$$

\section{Appendix 2 Probabilities of miss detection and false alarm of the CD and the FD}

To calculate $P\left[E_{n}>\lambda\right]$, there are two scenarios of the transmitted signals to be considered.

(1) A PS symbol is transmitted. In this case, the received sample $r_{n+k}$ follows a Gaussian distribution with mean $s_{n+k}$. In Equation 7, if $r_{n+2 k}$ and $s_{n+2 k}$ have the same sign, $\mathrm{C}_{\mathrm{r}, \mathrm{n}+2 \mathrm{k}} \mathrm{C}_{\mathrm{c}, 2 \mathrm{k}}^{*}=1$, else $\mathrm{C}_{\mathrm{r}, \mathrm{n}+2 \mathrm{k}} \mathrm{C}_{\mathrm{c}, 2 \mathrm{k}}^{*}=-1$. In this case, let $P_{4, \lambda}=P\left[E_{n}>\lambda\right]$. The same as the derivation of $P_{1, \lambda}$ in Appendix 1, $P_{4, \lambda}$ is given by

$$
P_{4, \lambda} \approx 1-\Phi\left(\frac{\frac{N / 2+\lambda}{4}-\frac{1}{2} \operatorname{erfc}\left(\frac{-\bar{a}}{\sqrt{2} \sigma^{2}}\right) N / 2}{\sqrt{\frac{1}{2} \operatorname{erfc}\left(\frac{-\bar{a}}{\sqrt{2} \sigma^{2}}\right)\left[1-\frac{1}{2} \operatorname{erfc}\left(\frac{-\bar{a}}{\sqrt{2} \sigma^{2}}\right)\right] N / 2}}\right)
$$

where $\bar{a}$ is the mean of the absolute values of the even coefficients. Because N/2 samples are used for calculating $E_{n}$, and not $\{0,1\}$ of the Bernoulli distribution but $\{-1,1\}$ is the value domain for $C_{r, n+2 k} C_{c, 2 k}^{*}$, instead of $\lambda, \frac{N / 2+\lambda}{2}$ is used in (31).

(2) Noise is transmitted. In this case, the received samples are Gaussian noise with zero mean. In this case, let $P_{5, \lambda}=P\left[E_{n}>\lambda\right]$. The same as the derivation of $P_{2, \lambda}$ in Appendix $1, P_{5, \lambda}$ is given by

$$
P_{5, \lambda} \approx 1-\Phi\left(\frac{\frac{N / 2+\lambda}{2}-\frac{1}{4} N}{\frac{1}{2} \sqrt{N / 2}}\right)
$$

In the $\mathrm{CD}, U_{n}$ is used to detect the received samples by comparing it with the predefined threshold. We assume that $\lambda$ is the threshold used for the CD. If PS symbols are present in both segments and $U_{n}<\lambda$, miss detection occurs. As $N$ samples are used for calculating $U_{n}$, according to (31), $P\left[U_{n}>\lambda\right]$ in this case is given by

$$
P\left[U_{n}>\lambda\right] \approx 1-\Phi\left(\frac{\frac{N+\lambda}{2}-\frac{1}{2} \operatorname{erfc}\left(\frac{-\bar{a}}{\sqrt{2} \sigma^{2}}\right) N}{\sqrt{\frac{1}{2} \operatorname{erfc}\left(\frac{-\bar{a}}{\sqrt{2} \sigma^{2}}\right)\left[1-\frac{1}{2} \operatorname{erfc}\left(\frac{-\bar{a}}{\sqrt{2} \sigma^{2}}\right)\right] N}}\right)
$$

Therefore, the miss detection probability of the $\mathrm{CD}$ is given by

$$
\begin{aligned}
& P_{c \_m}=1-P\left[U_{n}>\lambda\right] \\
& \approx \Phi\left(\frac{\frac{N+\lambda}{2}-\frac{N}{2} \operatorname{erfc}\left(\frac{-\bar{a}}{\sqrt{2} \sigma^{2}}\right)}{\sqrt{\frac{N}{2} \operatorname{erfc}\left(\frac{-\bar{a}}{\sqrt{2} \sigma^{2}}\right)\left[1-\frac{1}{2} \operatorname{erfc}\left(\frac{-\bar{a}}{\sqrt{2} \sigma^{2}}\right)\right]}}\right)
\end{aligned}
$$

If $U_{n}>\lambda$ and no PS symbol is present in either segment, false alarm occurs. According to (32), in this case,

$$
P_{c_{-} f}=P\left[U_{n}>\lambda\right] \approx 1-\Phi\left(\frac{\frac{N+\lambda}{2}-\frac{1}{2} N}{\frac{1}{2} \sqrt{N}}\right)
$$

The discrete value domain of $I_{n+k}\left|Q_{c, k}\right|$ of the PSMF in the FD is simplified as that in Appendix 1, and we assume that $M=2$ is selected for the FD and $\lambda$ is the predefined threshold.

If $D_{n}<\lambda$ and PS symbols are present in both segments, miss detection occurs.

$$
P_{f_{-} m}=1-P\left[D_{n}>\lambda\right]
$$

When $M=2$, a total of $2 N$ samples are used for calculating $D_{n}$. According to (26) in Appendix 1,

$$
P_{f_{-} m} \approx \Phi\left(\frac{\frac{N+\lambda}{2}-\operatorname{erfc}\left(\frac{-\bar{m}}{\sqrt{2} \sigma^{2}}\right) N}{\sqrt{\operatorname{erfc}\left(\frac{-\bar{m}}{\sqrt{2} \sigma^{2}}\right)\left[1-\frac{1}{2} \operatorname{erfc}\left(\frac{-\bar{m}}{\sqrt{2} \sigma^{2}}\right)\right] N}}\right)
$$

If $D_{n}>\lambda$ and no PS symbol is present in either segments, false alarm occurs. According to (29) in Appendix 1,

$$
P_{f_{-} f} \approx 1-\Phi\left(\frac{\frac{2 N+\lambda}{2}-N}{\frac{1}{2} \sqrt{2 N}}\right)
$$

\section{Abbreviations}

AC: auto-correlation; BLUE: best linear unbiased estimation; CC: crosscorrelation; CE: channel estimation; CD: coarse detector; CFO: carrier frequency offset; CIR: channel impulse response; FCC: Federal Communications Commission; FD: final detector; FS: frame synchronization; ICI: inter-carrier interference; IR: impulse radio; ISI: inter-symbol interference; MB-OFDM: multi-band orthogonal frequency-division multiplexing; PS: packet synchronization; PSD: power spectral density; QoS: quality of service; SD: signal detector; TFC: time-frequency code; TTD: TFC-type detector; UWB: ultra wideband; VLSI: very large scale integrated circuits; WPAN: wireless personal area network.

\section{Competing interests}

The authors declare that they have no competing interests.

Received: 15 January 2014 Accepted: 7 January 2015 Published online: 28 January 2015

\section{References}

1. Multiband OFDM Alliance SIG, Multiband OFDM physical layer proposal for IEEE 802.15 Task Group 3a, (2004) 
2. ECMA-368, High rate ultra wideband PHY and MAC standard, 3rd education (2008)

3. H Steendam, M Moeneclaey, Synchronization sensitivity of multi-carrier systems. Eur. Trans. Commun. 15(3), 223-234 (2004)

4. TM Schmidl, DC Cox, Robust frequency and timing synchronization for OFDM. IEEE Trans. Commun. 45(12), 1613-1621 (1997)

5. V Krishnamurthy, CRN Athaudage, H Dawei, Adaptive OFDM synchronization algorithms based on discrete stochastic approximation. IEEE Trans. Signal Process. 53(4), 1561-1574 (2005)

6. F Alessio, S Semih, OFDM symbol synchronization using frequency domain pilots in time domain. IEEE Trans. Wirel. Commun. 8(6), 3240-3248 (2009)

7. A Al-Dweik, S Younis, A Hazmi, C Tsimenidis, B Sharif, Efficient OFDM symbol timing estimator using power difference measurements. IEEE Trans. Vel. Technol. 61(2), 509-520 (2012)

8. S-W Choi, S-S Choi, A method of detecting time-frequency code in MB-OFDM UWB system, 2007 IEEE International Symposium on Consumer Electronics. Dallas 20-23, 1-4 (2007)

9. Z Ye, C Duan, PV Orlik, J Zhang, AA Abouzeid, A synchronization design for UWB-based wireless multimedia systems. IEEE Trans. Broadcast. 56(2), 211-225 (2010)

10. B.-H. Kim, C.-H. Shin, S. Choi, Packet detection scheme using cross correlation and complex multiplication in MB-OFDM UWB system, 2007 6th International Conference on Information, Communications \& Signal Processing, Zhengzhou China, December 2007, pp. 1-5

11. L Jyh-Ting, W An-Yeu, C Wen-Chiang, A systematic design approach to the band-tracking packet detector in OFDM-based ultrawideband systems. IEEE Trans. Vehic. Techn. 56(6), 3791-3806 (2007)

12. W Fan, C-S Choy, L Ka Nang, Robust and low complexity packet detector design for MB-OFDM UWB system, 2009 IEEE International Symposium on Circuits and Systems. Taipei 24-27, 693-696 (2009)

13. L Zhang, EKS Au, VKN Lau, A three-stage cross correlation-based timing synchronization algorithm for MB-OFDM UWB systems, 2008 11th IEEE Singapore International Conference on Communication Systems. Poland 19-21, 1534-1538 (2008)

14. F Wen, C Chiu-Sing, Robust, low-complexity, and energy efficient downlink baseband receiver design for MB-OFDM UWB system. IEEE Trans. Circuits. Syst. 59(2), 399-408 (2012)

15. CW YAK, Z Lei, S Chattong, TT Tjhung, Timing synchronization for ultra-wideband (UWB) multi-band OFDM systems, 2005 IEEE 62nd Vehicular Technology Conference. Dallas 25-28, 1599-1603 (2005)

16. L Yinghui, $M$ Hlaing, J Trent, MZ Win, Frequency offset estimation for MB-OFDM-based UWB systems. IEEE Trans. Commu. 56(6), 4729-4734 (2008)

17. X. Wang, Z. Qian, Y. Wang, A polarity comparison timing synchronization estimation for MB-OFDM-based UWB systems. EURASIP J. Wirel. Commun. Netw. (2012). doi:10.1186 /1687-1499-2012-169

18. AM Karim, O Masuri, Improved fine CFO synchronization for MB-OFDM UWB. IEEE Commu. Lett. 14(4), 351-353 (2010)

\section{Submit your manuscript to a SpringerOpen ${ }^{\odot}$ journal and benefit from:}

- Convenient online submission

- Rigorous peer review

- Immediate publication on acceptance

- Open access: articles freely available online

- High visibility within the field

- Retaining the copyright to your article

Submit your next manuscript at $>$ springeropen.com 